\title{
1 Dose-dependent role of Gfi1 in murine hematopoietic stem cell self-renewal
}

\section{2 and differentiation}

3 Judith Schütte ${ }^{1,2}$, Aniththa Thivakaran ${ }^{1}$, Yahya Al-Matary ${ }^{1,2}$, Pradeep Kumar Patnana ${ }^{1,2}$,

$4 \quad$ Daria Frank ${ }^{1,2}$, Daniel R. Engel ${ }^{3}$, Ulrich Dührsen ${ }^{1}$, Cyrus Khandanpour ${ }^{1,2, *}$

5

6 Universitätsklinikum Essen, Klinik für Hämatologie, WTZ-Forschung, Hufelandstr. 55, 45147 Essen,

7 Germany

$8 \quad{ }^{2}$ Present address: Universitätsklinikum Münster, Medizinische Klinik A, Albert-Schweitzer-Campus

9 1, Gebäude D3, 48149 Münster, Germany

10 Universitätsklinikum Essen, Institut für Experimentelle Immunologie und Bildgebung, Abteilung für

11 Immundynamik, Hufelandstr. 55, 45147 Essen, Germany

12

* Corresponding author

Corresponding author:

PD Dr. med. Cyrus Khandanpour,

48149 Münster,

Phone: +49 251 / 8346012

Email: cyrus.khandanpour@ukmuenster.de 


\section{Abstract}

Gfil (Growth factor independence 1) is a transcription factor that influences the stem cell capacity of hematopoietic stem cells (HSCs) as well as their differentiation into the myeloid and lymphoid lineage. Loss of Gfil impedes the repopulation capacity of HSCs and leads to a block in granulocyte generation causing severe neutropenia and monocytosis. Competitive transplantation assays showed that Gfil-deficient cells were not able to reconstitute myeloid and lymphoid hematopoiesis in competition with Gfil-wildtype (GFI1-36S) cells. Low Gfil levels $(G F I 1$-knockdown $=G F I 1-\mathrm{KD})$ in blasts of myelodysplastic neoplasms, acute and chronic myeloid leukemia patients are associated with poor patient survival. To understand how reduced levels or loss of Gfil contribute to hematopoiesis, we analyzed the effect of GFII-KD and Gfil-KO on HSCs and more mature cell types in mice. GFI1-KD and Gfil-KO led to strong decrease in HSC numbers, while the numbers of early progenitors ( $\mathrm{Lin}^{-}$ $\mathrm{Scal}^{+} \mathrm{cKit}^{+}$cells) were slightly increased. Competitive transplantation assays showed that GFII-KD and Gfil-KO HSCs can still engraft and expand, but they cannot contribute to myeloid and lymphoid differentiation. 


\section{Introduction}

Gfil (Growth factor independence 1) is a transcriptional repressor with a crucial role in hematopoietic stem cells (HSCs) as well as more mature cells of the myeloid and lymphoid lineage $(1,2)$. It regulates maturation of myeloid cells and is required for different signaling pathways such as Notch signaling (3-5). Analysis of Gfil expression in Gfil:GFP heterozygous knock-in mice revealed that Gfil is primarily expressed in HSCs, GMPs (granulocyte-macrophage progenitors) and CLPs (common lymphoid progenitors), while it is absent in CMPs (common myeloid progenitors) and MEPs (megakaryocyte-erythroid progenitors) (6). Gfil-deficient mice are characterized by an accumulation of monocytic cells and absence of granulocytes, leading to severe neutropenia and monocytosis $(7,8)$. Recently it has been implicated that reduced levels of Gfil are associated with an inferior prognosis for human MPN (myeloproliferative neoplasm), CML (chronic myeloid leukemia) and AML (acute myeloid leukemia) patients and with an acceleration of AML development in mice (911). These studies also showed that Gfil regulates expression of certain oncogenes, apoptotic pathways and possibly metabolic functions in a dose-dependent manner. However, it has also been postulated that loss of Gfil negatively influences the repopulation capacity of HSCs (6, 12).

We were now interested whether low levels and loss of Gfil indeed negatively affect the stem cell and differentiation capacity of HSCs and how the seemingly contradictory findings between reduced self-renewal capacity and the dose-dependent role of Gfil function in myeloid pathogenesis could be reconciled. For the analysis we made use of Gfil-knockout (KO) mice, a mouse strain with a complete loss of Gfil, and GFI1-knockdown (KD) mice, a mouse strain in which we cloned the human GFIl sequence into the murine Gfil gene locus together with a Neo cassette in the opposite direction of transcription, leading to GFII expression of only 10-15\% of wild-type levels (10). We show here that GFI1-KD and Gfil- 
62

63

64

65

66

67

68

69

$\mathrm{KO}$ mice contain a higher number of hematopoietic progenitors (LSK cells, $\mathrm{Lin}^{-} \mathrm{Scal}^{+} \mathrm{c}-\mathrm{Kit}^{+}$), but $\mathrm{HSC}\left(\mathrm{Lin}^{-} \mathrm{Sca}{ }^{+} \mathrm{c}-\mathrm{Kit}^{+} \mathrm{CD} 150^{+} \mathrm{CD} 48^{-}\right)$numbers are strongly reduced. Although GFI1-KD and Gfil-KO mice have fewer HSCs compared to Gfil-WT mice, these HSCs can still engraft and expand in a competitive setting. However, they have lost their ability to contribute to multi-lineage differentiation.

\section{Methods}

Mice

GFI1-36S, Gfil-KO and GFII-KD mice have previously been described (8, 10, 13). Mice were housed in specific pathogen-free conditions at the animal facility of University Hospital Essen, Germany. All experiments were approved by the local authorities (LANUV, Az8402.04.2015.A022).

Mice were irradiated using the X-Rad320 device from Precision X-ray. GFP-sorted whole $\mathrm{BM}$ cells or Lineage negative cells were transplanted intravenously.

\section{Lineage negative cell isolation}

$\mathrm{BM}$ cells were isolated from tibiae, femora and humeri and red blood cells were removed by using BD Pharm Lyse ${ }^{\mathrm{TM}}$ (555899, BD Biosciences). Lineage negative cells were collected by applying the Direct Lineage Cell Depletion Kit mouse (130-090-858, Miltenyi Biotec $\mathrm{GmbH}$ ) and magnetic separation following manufacturer's instructions. 
84

The following antibodies were used to differentiate the various cell types: Lineage Cell Detection Cocktail-Biotin, mouse (130-092-613) from Miltenyi Biotec GmbH, FITC antimouse Ly-6A/E (Sca-1) (108105), Brilliant Violet 421 (105827), PerCP/Cy5.5 anti-mouse CD45.1 (110728), PE anti-mouse CD45.2 (109808), PE/Cy7 anti-mouse CD150 (SLAM) (115913), APC anti-mouse CD48 (103411), APC antimouse/human CD11b (101211), APC anti-mouse CD8a (100711), FITC anti-mouse CD4 (100509) and FITC anti-mouse/human CD45R/B220 (103205) from BioLegend, Streptavidin PerCP-Cyanine5.5 (45-4317-82) and Ly-6G/Ly-6C Monoclonal Antibody (RB6-8C5) FITC from eBioscience. Cells were analysed using a BD LSR II device from BD Biosciences.

Statistical Analysis

Analyses were performed using Microsoft Excel. Significance was calculated using a paired ttest with a normal distribution.

All methods were performed in accordance with the relevant guidelines and regulations.

\section{Results and Discussion}

GFI1 influences prognosis of AML, CML and MPN patients in a dose-dependent manner, and in murine models of human leukemia reduced levels of GFI1 accelerated disease development (9-11, 14). AML, CML and MPN arise as a result of transformation of HSCs or early hematopoietic progenitors that still possess some degree of stem cell capacity (15). It has been described that complete loss of Gfil leads to an impaired function of HSCs $(6,7)$ and an increase in LSK frequency, possibly due to an increased proliferation $(7,16)$. It has previously 
been reported that Gfil-KO cells fail to compete with Gfil-WT cells in a competitive setting, demonstrated by a lack of reconstitution capacity of the myeloid and lymphoid compartment in the peripheral blood (PB) (6). The question arising from these results is whether the lack of mature cells in a competitive transplantation setting is a) due to a failure of Gfil-KO HSCs to engraft, b) due to a lack of survival of Gfil-KO HSCs in competition with Gfil-WT HSCs or c) due to a loss of Gfil-KO HSC capacity to contribute to PB cell production. We were hence interested to determine whether reduced levels or loss of GFI1/Gfil influence a) the number of HSCs and b) their ability to engraft and reconstitute the PB and BM of recipient mice in a competitive transplantation setting.

To this end, we made use of the previously described GFI1-KD mice which express human GFI1 at around 10-15\% of normal GFI1 levels (10) and Gfil-KO mice with a complete loss of Gfil expression (8). As a control we used GFI1-36S mice, a mouse strain containing the human GFIl cDNA sequence at the position of the murine Gfil locus, as this is the mouse strain from which GFI1-KD mice were derived. We have shown in the past that GFI1-36S mice are functionally equivalent to murine $G f i 1-W T$ mice $(10,13,17,18)$. In addition, they do not differ in their number of hematopoietic stem and progenitor cells (Supplementary Figure 1

$\mathrm{A}$ and B). We first examined the frequency of hematopoietic progenitor cells in GFI1-36S, GFI1-KD and Gfil-KO mice. While the percentage of $\mathrm{LinS}^{-} \mathrm{Scal}{ }^{+} \mathrm{c}-\mathrm{Kit}^{+}$(LSK) cells was significantly increased in GFII-KD mice compared to GFII-36S mice, the number of LSKs in Gfil-KO mice was only marginally elevated (Fig. 1A and B). In contrast, the percentage of HSCs defined as $\mathrm{Lin}^{-} \mathrm{Sca}^{+} \mathrm{c}-\mathrm{Kit}^{+} \mathrm{CD} 150^{+} \mathrm{CD} 48^{-}$was highly reduced in both, GFII-KD and Gfil-KO mice (Fig. $1 \mathrm{~A}$ and C). This observation was irrespective of the age of the mice (Supplementary Fig. $1 \mathrm{C}$ and D). Our data thus recapitulate previous findings of our and other groups regarding an expansion of LSKs upon loss of GFII (7, 16), but further expands our knowledge about a dose-dependent effect of GFII expression on HSCs, namely a statistically significant, stepwise decrease in HSC numbers. 
We next investigated how reduced levels or loss of GFI1 in HSCs affect their ability to engraft after transplantation and to differentiate into the various cell lineages. To study this we performed a competitive bone marrow (BM) transplantation assay (Fig. 2A). We isolated Lin ${ }^{-}$ cells from CD45.1-expressing Gfil-WT mice and mixed those cells in equal numbers with Lin $^{-}$cells from CD45.2-expressing GFI1-KD or Gfil-KO mice. Next, we transplanted a total of $10^{5} \mathrm{Lin}^{-}$cells into lethally irradiated C57BL/6 mice and analysed the PB and BM for their CD45.1 and CD45.2 expression. Although GFI1-KD and Gfil-KO mice contain fewer HSCs than Gfil-WT mice, we transplanted equal numbers of $\mathrm{Lin}^{-}$cells, the cell fraction that contains hematopoietic stem and progenitor cells. We chose to transplant Lin $^{-}$cells and not phenotypically more defined HSCs because it has recently been shown that altered transcription factor levels can influence which cell fractions give rise to long-term repopulation. The combined deletion of $p 16^{\operatorname{Ink} 4 a}, p 19^{A r f}$ and $\operatorname{Trp53}$ in mice for instance led to the acquisition of stem-cell-like properties by multipotent progenitors (19). Repeating the transplantation experiments with highly purified HSCs was not allowed due to animal regulations demanding a reduction of mouse numbers. Transplantation of $\operatorname{Lin}^{-}$cells thus results in a potential bias towards transplantation of fewer GFI1-KD or Gfil-KO HSCs (based on surface marker expression) compared to Gfil-WT HSCs, but ensures that all functional cells are included in the transplanted cell fraction. To determine the contribution of Gfil-WT or GFI1-KD/Gfil-KO HSCs to hematopoiesis, we analyzed not only the overall presence of $\mathrm{CD} 45.2^{+}$cells in $\mathrm{PB}$, but also the presence of short-lived $\mathrm{CD} 45.2^{+}$myeloid cells such as monocytes over time. The presence of monocytes derived from CD45.2 $2^{+}$GFI1-KD or Gfil$\mathrm{KO}$ cells in $\mathrm{PB}$ more than 6 weeks after transplantation rules out a potential carry-over of these cells as a result of the transplantation. To check whether GFI1-KD and Gfil-KO derived cells possess HSC activity, we determined the presence of short-lived monocytes in PB. transplantation, respectively, hence originated from GFI1-KD HSCs in the competitive 
transplantation assay (Fig. 2 B). As it has previously been shown that long-term reconstitution can be determined 12-16 weeks after transplantation $(19,20)$, this result indicates that GFIIKD HSCs possess indeed long-term HSC activity and that GFI1 has a dose-dependent effect in contribution to peripheral blood cell formation. In contrast, CD45.2-derived monocytes were not present in the Gfil-KO transplantation experiment, even after 21 days of transplantation (Fig. 2 C). This finding is expected as it has been shown in previous experiments that Gfil-KO cells do not contribute to mature cells in $\mathrm{PB}$ in a competitive transplantation setting $(6,12)$. In addition to monocytes, we also measured the overall contribution of CD45.2 transplanted cells to the PB composition. On day 308 after transplantation of GFII-KD cells together with Gfil-WT cells, almost $90 \%$ of all cells in the PB had a CD45.1 origin (Fig. 2B). A very similar pattern could be observed 112 days after transplantation of Gfil-KO cells together with Gfil-WT cells (Fig. 2C). Once CD45.2-derived short-lived monocytes were not detected anymore in PB (after 308 days for GFII-KD and after 112 days for Gfil-KO), we stopped the experiment and analyzed the BM for presence of HSCs. Interestingly, two out of four transplanted mice that were generated from CD45.2expressing GFI1-KD cells contained a high percentage of HSCs in the BM (44 and $88 \%$ respectively), while the HSCs of the other two mice originated from CD45.1-expressing GfilWT cells (Fig. 2D). Similarly, 25-40\% of HSCs arose from CD45.2-expressing cells in three out of four mice transplanted with equal numbers of Gfil-KO and Gfil-WT cells, thus arising from Gfil-KO cells (Fig. 2E). In contrast to the origin of HSCs, most of the mature cell types of the PB developed from CD45.1-expressing Gfil-WT cells at 308 days in GFI1-KD or 112 days in Gfil-KO competitive transplantation experiments (Fig. 2F and G). At day 308 after transplantation, $0.3 \%$ of the $\mathrm{Ly}_{6 \mathrm{G}} \mathrm{CD}^{\mathrm{hi}} 11 \mathrm{~b}^{+}$(granulocytes) and $1.2 \%$ of $\mathrm{Ly} 6 \mathrm{G}^{\mathrm{int}} \mathrm{CD} 11 \mathrm{~b}^{+}$ (monocytes) originated from GFI1-KD HSCs. This lack of contribution of HSCs to more committed cells was already observable at an early timepoint of the hematopoietic hierarchy as the majority of LSKs in both competitive transplantation experiments were CD45.2- 
negative (Supplementary Fig. 2). We also examined the contribution of HSC to other compartments. After 308 days, CD45.2-expressing GFI1-KD cells still contributed to $4 \%$ of all $\mathrm{CD}^{+}$cells, $13.5 \%$ of all $\mathrm{CD}^{+}$cells and $7.5 \%$ of all $\mathrm{B} 220^{+}$cells in $\mathrm{PB}$ (Fig. $2 \mathrm{~F}$ ). While $\mathrm{B} 220^{+}$cells only very rarely arised from CD45.2-expressing Gfil-KO cells, these cells still contributed to $12 \%$ of all $\mathrm{CD}^{+}$and $23 \%$ of all $\mathrm{CD}^{+}$cells 112 days after transplantation (Fig. 2G). It is more difficult to estimate the contribution of transplanted HSCs to the cells of the lymphoid lineage, as these cell types are generally long-lived. They could thus originate from the host. Alternatively, if they were generated from the transplanted HSCs early after transplantation, these cells would still be detectable at later time-points, even though HSCs do not self-renew and differentiate anymore. It can also not be ruled out that these cells arise from more committed progenitor cells which were co-transplanted with the Lin cells.

In summary, we expand our knowledge about the role of Gfil in HSCs by showing that reduced levels and loss of Gfil lead to a slight expansion of LSK cells, but to highly significant reduction of HSCs, and that this reduction is dependent on the Gfil level. On a functional level, reduced expression or loss of GFII expression did not dramatically impede the ability of GFII-KD or Gfil-KO HSCs to engraft or to expand after transplantation (up to $80 \%$ of HSCs were of GFII-KD origin and up to $40 \%$ of HSCs were of Gfil-KO origin), but negatively and in a dose-dependent manner influenced the maturation of HSCs into the lymphoid and myeloid lineages. Our data are in line with other reports highlighting the role of Gfil in lineage choice, similar to that of other transcription factors such as IRF8 and PU.1 $(21,22)$. Our arising hypotheses are that a) knockdown and loss of GFI1 might regulate the choice of symmetric and asymmetric division of HSCs which could explain the leukemiapropagating function of reduced GFI1 levels in CML and AML (10) or b) GFI1-KD or GfilKO HSCs exhaust their ability to produce mature cells after a certain time point. As Gfil-KO cells have a normal life span in a non-competitive setting in mice if they are kept under SPF 
210 conditions (23), further experiments are needed to study the dose-dependent role of Gfil in

211 HSC function.

214 The authors declare no competing interests.

217 The authors would like to thank Marina Suslo and Renata Köster for excellent technical

218 assistance and the team of the animal facility at University Hospital Essen for genotyping, 219 technical and administrative assistance during the whole mouse project. J.S. and the work 220 were supported by a grant of the Fritz-Thyssen foundation. C.K. was supported by the 221 IFORES fellowship of the University Hospital Essen, a Max-Eder fellowship from the 222 German Cancer Fund (Deutsche Krebshilfe) as well as from the Dr. Werner Jackstädt223 Stiftung.

J.S. performed experiments and wrote the paper. A.T., Y.A.-M., P.K.P, D.F. performed the project. 
1. van der Meer LT, Jansen JH, van der Reijden BA. Gfi1 and Gfilb: key regulators of hematopoiesis. Leukemia. 2010;24(11):1834-43.

2. Phelan JD, Shroyer NF, Cook T, Gebelein B, Grimes HL. Gfi1-cells and circuits: unraveling transcriptional networks of development and disease. Current opinion in hematology. 2010;17(4):300-7.

3. Horman SR, Velu CS, Chaubey A, Bourdeau T, Zhu J, Paul WE, et al. Gfi1 integrates progenitor versus granulocytic transcriptional programming. Blood. 2009;113(22):5466-75.

4. Phelan JD, Saba I, Zeng H, Kosan C, Messer MS, Olsson HA, et al. Growth factor independent1 maintains Notch1-dependent transcriptional programming of lymphoid precursors. PLoS genetics. 2013;9(9):e1003713.

5. Velu CS, Baktula AM, Grimes HL. Gfi1 regulates miR-21 and miR-196b to control myelopoiesis. Blood. 2009;113(19):4720-8.

6. Zeng H, Yucel R, Kosan C, Klein-Hitpass L, Moroy T. Transcription factor Gfi1 regulates selfrenewal and engraftment of hematopoietic stem cells. The EMBO journal. 2004;23(20):4116-25.

7. Hock H, Hamblen MJ, Rooke HM, Traver D, Bronson RT, Cameron S, et al. Intrinsic requirement for zinc finger transcription factor Gfi-1 in neutrophil differentiation. Immunity. 2003;18(1):109-20.

248 8. Karsunky H, Zeng H, Schmidt T, Zevnik B, Kluge R, Schmid KW, et al. Inflammatory reactions and severe neutropenia in mice lacking the transcriptional repressor Gfil. Nature genetics. 2002;30(3):295-300.

251 9. Fraszczak J, Vadnais C, Rashkovan M, Ross J, Beauchemin H, Chen R, et al. Reduced expression but not deficiency of GFI1 causes a fatal myeloproliferative disease in mice. Leukemia. 2018.

10. Hones JM, Botezatu L, Helness A, Vadnais C, Vassen L, Robert F, et al. GFI1 as a novel prognostic and therapeutic factor for AML/MDS. Leukemia. 2016;30(6):1237-45.

11. Kok CH, Watkins DB, Leclercq T, D'Andrea RJ, Hughes TP, White DL. Low GFI1 expression in white blood cells of $\mathrm{CP}-\mathrm{CML}$ patients at diagnosis is strongly associated with subsequent blastic transformation. Leukemia. 2013;27(6):1427-30. proliferation and preserves functional integrity of haematopoietic stem cells. Nature. 2004;431(7011):1002-7.

13. Khandanpour C, Krongold J, Schutte J, Bouwman F, Vassen L, Gaudreau MC, et al. The human GFI136N variant induces epigenetic changes at the Hoxa9 locus and accelerates K-RAS driven myeloproliferative disorder in mice. Blood. 2012;120(19):4006-17. significance of high GFI1 expression in AML of normal karyotype and its association with a FLT3-ITD signature. Scientific reports. 2017;7(1):11148. and GMP-like leukemia stem cells in acute myeloid leukemia. Cancer cell. 2011;19(1):138-52. independence 1 protects hematopoietic stem cells against apoptosis but also prevents the development of a myeloproliferative-like disease. Stem cells. 2011;29(2):376-85. expression impedes human and murine leukemic cell growth. Scientific reports. 2017;7(1):15720. 
18. Botezatu L, Michel LC, Helness A, Vadnais C, Makishima H, Hones JM, et al. Epigenetic therapy as a novel approach for GFI136N-associated murine/human AML. Experimental hematology. 2016;44(8):713-26.e14.

278 19. Akala OO, Park IK, Qian D, Pihalja M, Becker MW, Clarke MF. Long-term haematopoietic reconstitution by Trp53-/-p16Ink4a-/-p19Arf-/- multipotent progenitors. Nature. 2008;453(7192):228-32.

281 20. Morrison SJ, Weissman IL. The long-term repopulating subset of hematopoietic stem cells is deterministic and isolatable by phenotype. Immunity. 1994;1(8):661-73. 21. Olsson A, Venkatasubramanian M, Chaudhri VK, Aronow BJ, Salomonis N, Singh H, et al. Single-cell analysis of mixed-lineage states leading to a binary cell fate choice. Nature. 2016;537(7622):698-702. 22. Spooner CJ, Cheng JX, Pujadas E, Laslo P, Singh H. A recurrent network involving the transcription factors PU.1 and Gfi1 orchestrates innate and adaptive immune cell fates. Immunity. 2009;31(4):576-86. neutropenia and induces osteoporosis depending on the pathogen load and systemic inflammation. PLOS ONE. 2018;13(6):e0198510. 
HSCs. Murine BM cells were isolated and sorted for surface marker expression (lineage six mice were analyzed. Error bars represent standard deviation. $*=p$-value $\leq 0.05$. C.

Knockdown or loss of GFI1/Gfil resulted in a dose-dependent decrease of HSCs $\left(\mathrm{Lin}^{-} \mathrm{Sca} 1^{+} \mathrm{c}-\right.$ the percentage of HSCs compared to total BM cells. For each genotype six mice were analyzed. Error bars represent standard deviation. $* \leq 0.05, * *=\mathrm{p}$-value $\leq 0.01, * * *=\mathrm{p}$-value $\leq$

Figure 2: Gfil is essential for self-renewal of HSCs and their differentiation. A.

Schematic diagram of the competitive transplantation assay setup. Lin $^{-}$cells were isolated

mouse. The cells were mixed in equal numbers. A total of $10^{5}$ Lin $^{-}$cells were transplanted into lethally irradiated C57BL/6 mice. For each competitive transplantation assay four mice were transplanted ( $\mathrm{n}=4,1$ experiment). The $\mathrm{BM}$ and $\mathrm{PB}$ were analyzed for the presence of HSCs and mature blood cells at different time points. B. CD45 expression on the cell surface of all cells of the PB (PB) or more specific monocytes (MC) was measured by flow cytometry 33, 77, 97, 132 and 308 days after transplantation. C. CD45 expression on the cell surface of all cells of the PB or more specific monocytes (MC) was measured by flow cytometry 21 and 
were isolated 315 days (KP7819 and KP7920) or 322 days (KP7817 and KP7818) after transplantation. The origin of HSCs was analyzed by flow cytometry. E. CD45.2-expressing Gfil-KO cells contributed to the generation of HSCs $\left(\mathrm{Lin}^{-} \mathrm{Sca} 1^{+} \mathrm{c}-\mathrm{Kit}^{+} \mathrm{CD} 150^{+} \mathrm{CD}^{4} 8^{-}\right)$. Four mice with the indicated names (KP10388, KP10389, KP10390 and KP10391) were subjected to transplantation and BM cells were isolated 112 days after transplantation. The origin of HSCs was analyzed by flow cytometry. F. Almost all mature blood cells of the PB had a CD45.1-expressing Gfil-WT origin 308 days after transplantation of CD45.1-expressing Gfil-WT and CD45.2-expressing GFI1-KD cells. Granulocytes defined as Ly6G ${ }^{\text {hi }}$ CD11 ${ }^{+}$, Monocytes defined as $\mathrm{Ly}_{6 \mathrm{G}} \mathrm{G}^{\mathrm{int}} \mathrm{CD} 11 \mathrm{~b}^{+}$. Shown is the analysis of four mice. G. Almost all mature blood cells of the PB had a CD45.1-expressing Gfil-WT origin 112 days after transplantation of CD45.1-expressing Gfil-WT and CD45.2-expressing Gfil-KO cells. Granulocytes defined as Ly6G ${ }^{\text {hi }} \mathrm{CD} 11 b^{+}$, Monocytes defined as $\mathrm{Ly}_{6} \mathrm{G}^{\text {int }} \mathrm{CD} 11 b^{+}$. Shown is the analysis of four mice. Error bars for all sub-figures represent standard deviation.

\section{Supplementary Figure 1: A./B. Number of LSK $\left(\mathrm{Lin}^{-} \mathrm{c}-\mathrm{Kit}^{+} \mathrm{Sca} 1^{+}\right)$cells (A) and HSCs (B)} from Gfil-wt mice containing the murine Gfil gene are comparable with numbers of LSK cells or HSCs, respectively, from GFI1-36S mice in which the murine Gfil gene was replaced by the human GFII cDNA sequence. $\mathrm{n}=$ number of mice analyzed. No statistical difference detectable. C./D. The number of LSK cells (C) and HSCs (D) from GFI1-36S, GFI1-KD and Gfil-KO mice did not change with age. Young $=39-127$ days; old $=190-251$ days. No statistical difference detectable.

\section{Supplementary Figure 2: The differentiation defect is established early during} hematopoiesis. A. CD45.2-expressing GFI1-KD cells rarely contributed to $\mathrm{Lin}^{-} \mathrm{c}-\mathrm{Kit}^{+} \mathrm{Sca} 1^{+}$ (LSK) cell compartment. Four mice with the indicated names (KP7817, KP7818, KP7819 and KP7820) were subjected to transplantation and BM cells were isolated 315 days (KP7819 and 
345 KP7920) or 322 days (KP7817 and KP7818) after transplantation. Cells were analyzed by

346 flow cytometry. B. CD45.2-expressing Gfil-KO cells hardly contribute to the generation of

347 LSK cells. Four mice with the indicated names (KP10388, KP10389, KP10390 and KP10391)

348 were subjected to transplantation and BM cells were isolated 112 days after transplantation.

349 Cells were analyzed by flow cytometry. 
A
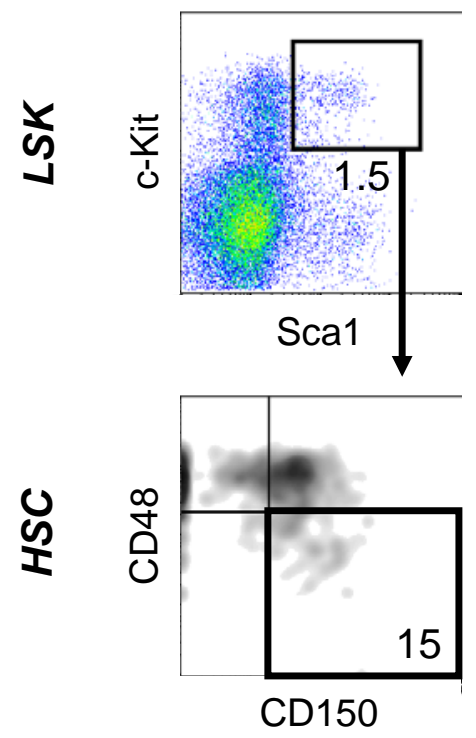

B

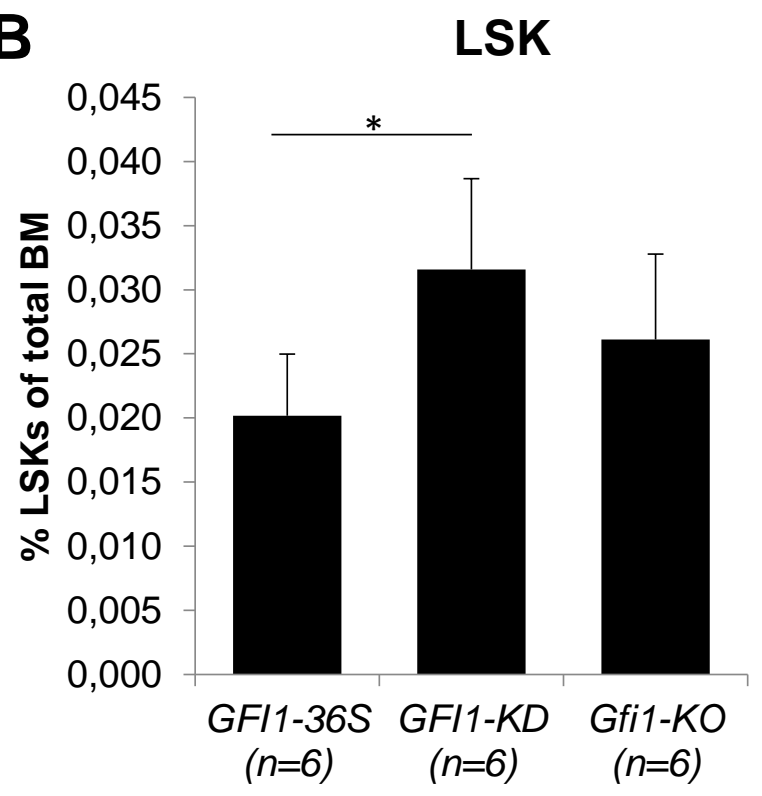

GFI1-KD
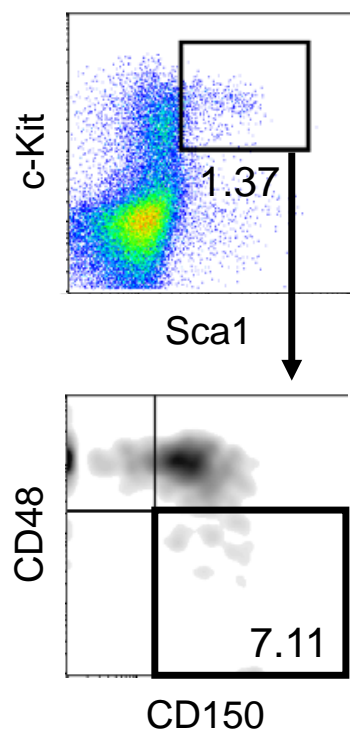

C
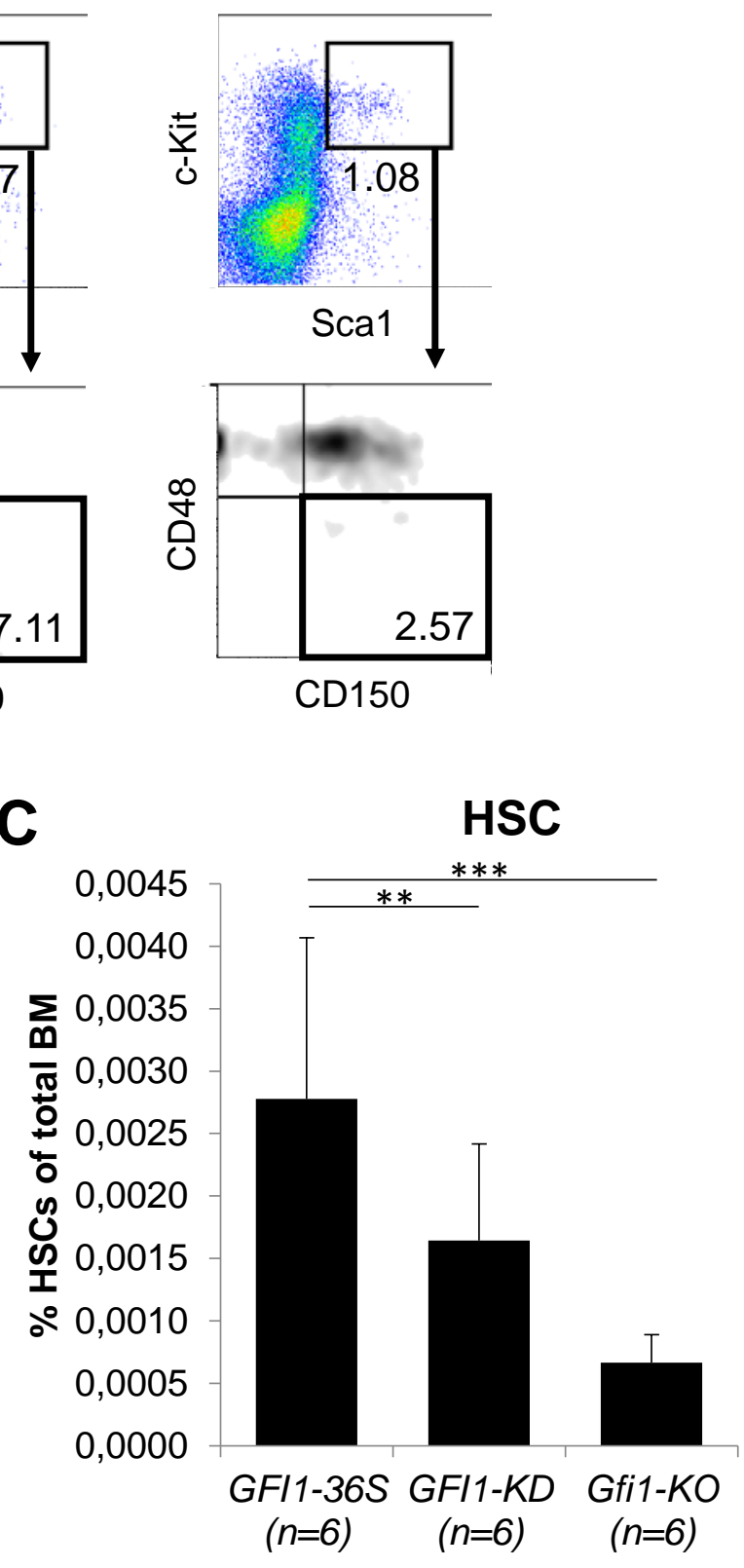


\section{Figure 2}

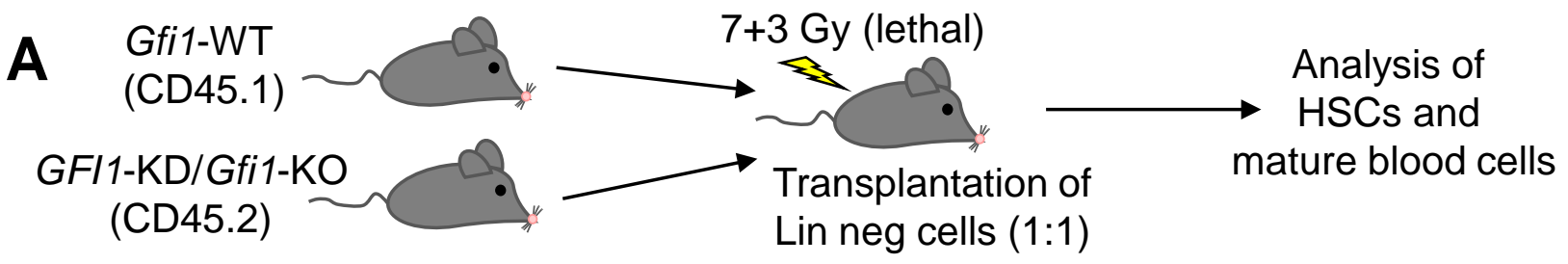

GFI1-KD

B

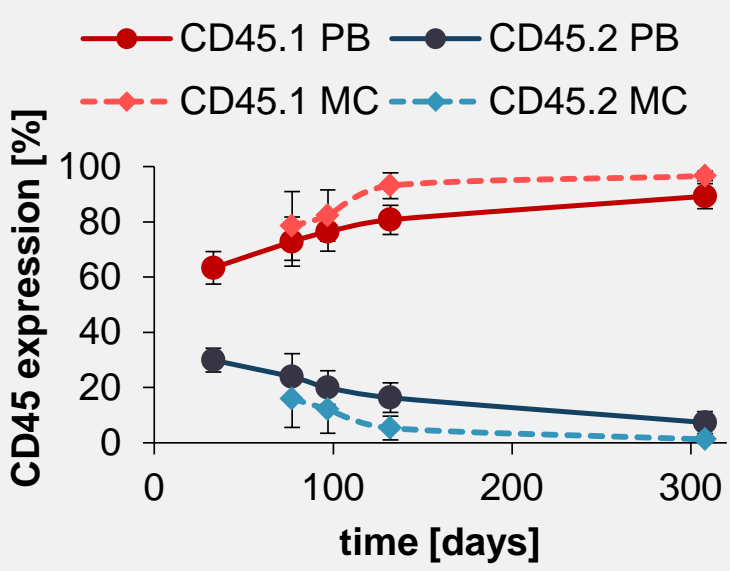

D

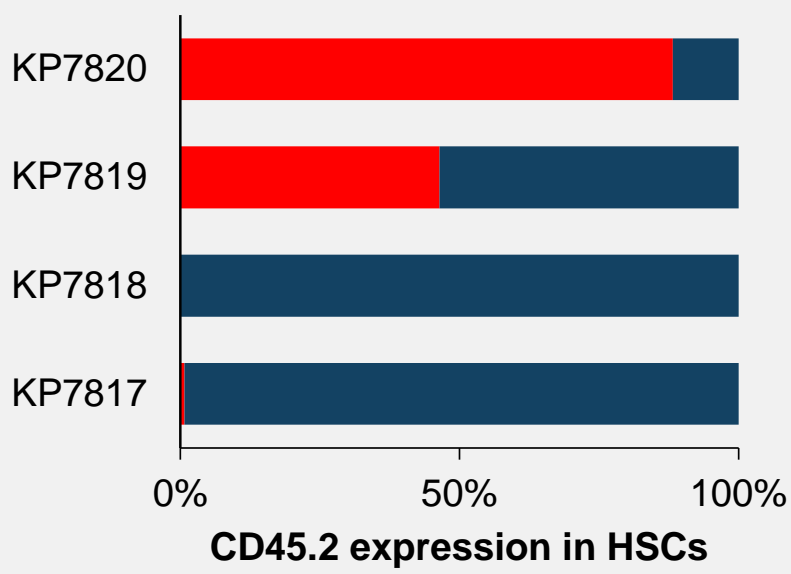

F

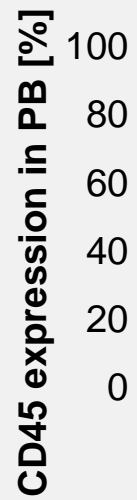

-CD45.1 CD45.2

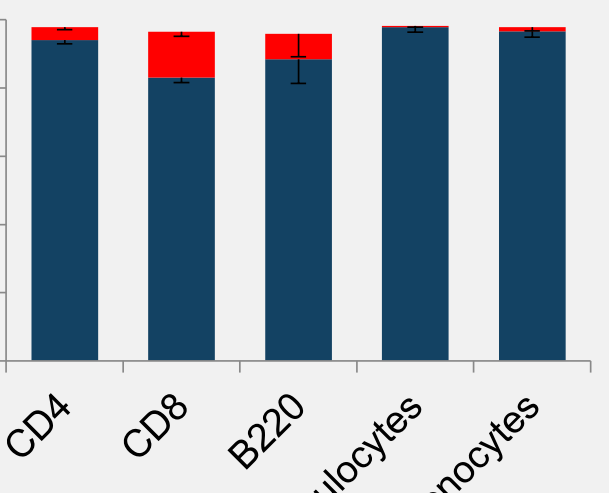

C

Gfi1-KO

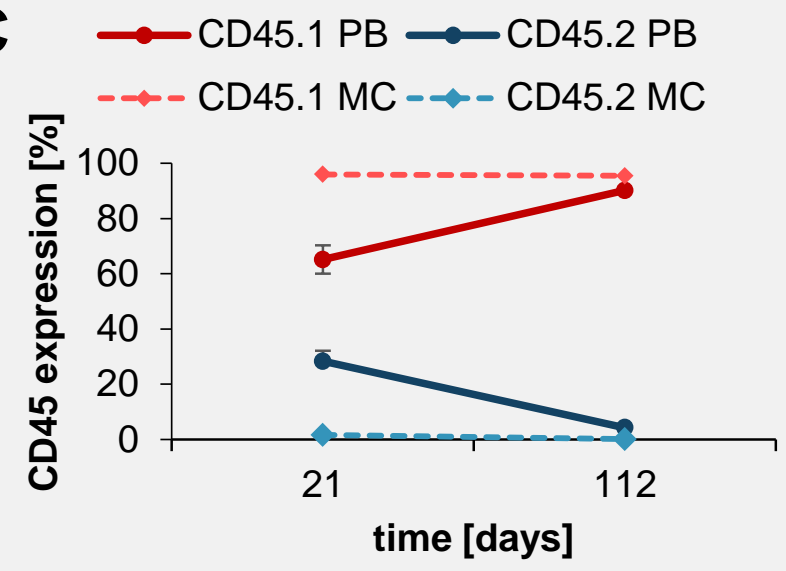

E

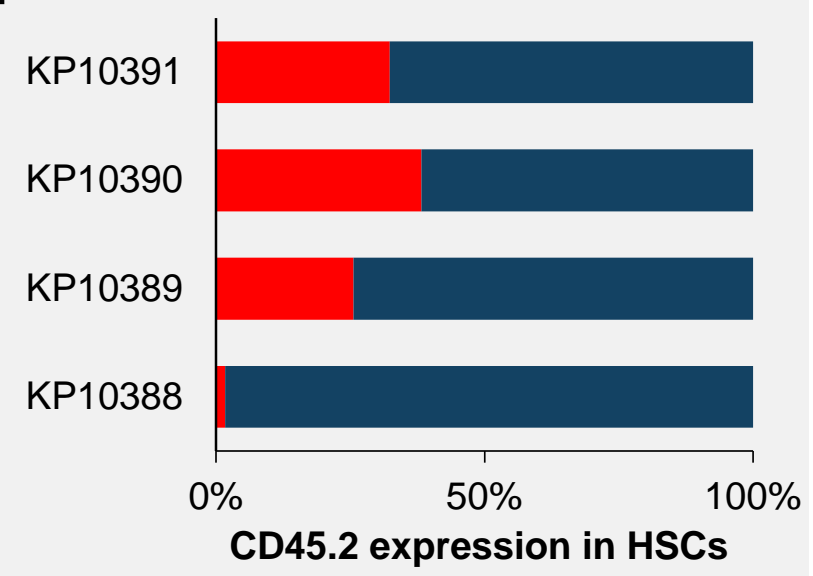

G

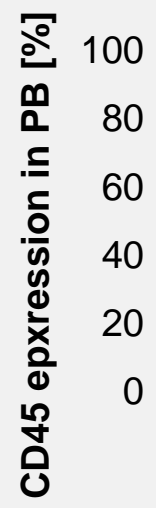

- CD45.1 - CD45.2

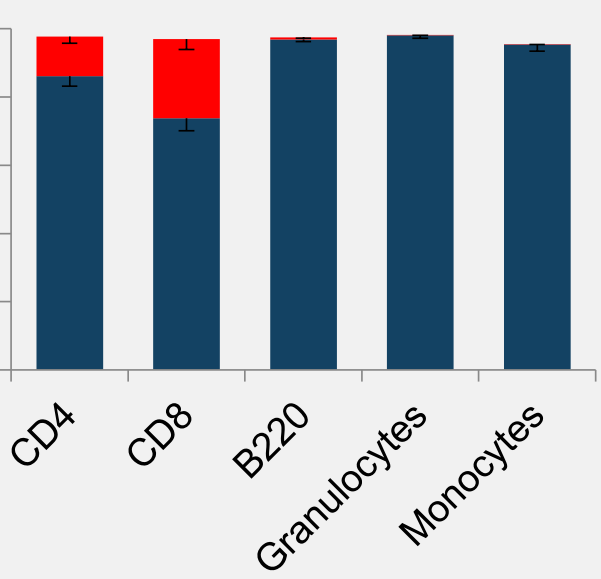


Supplementary figure 1

A

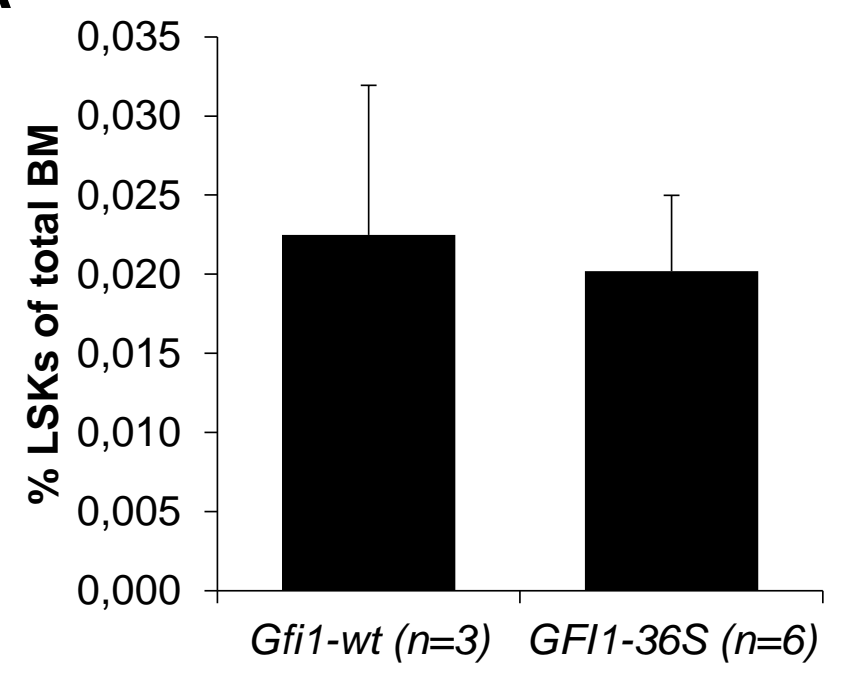

C

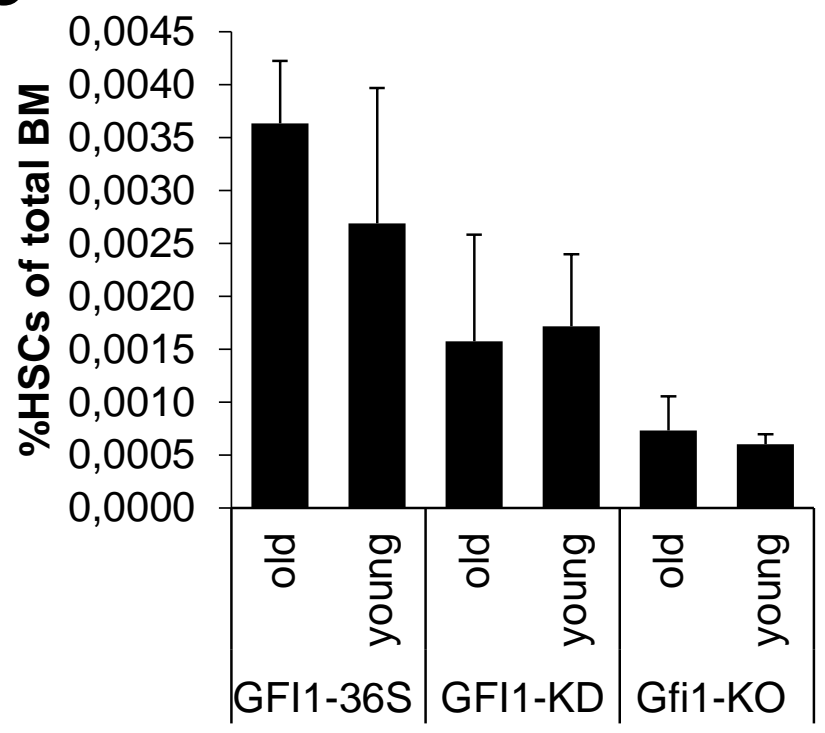

B

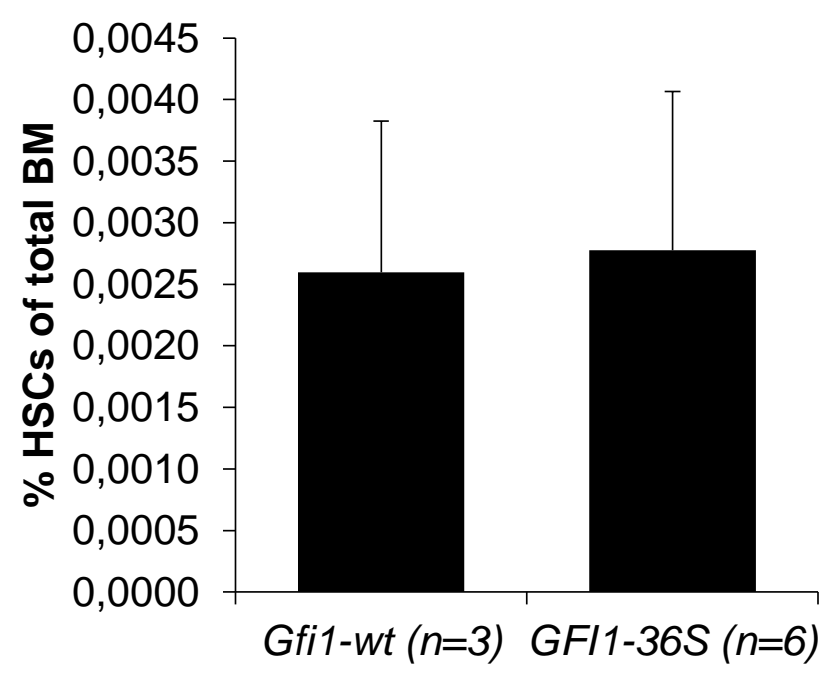

D

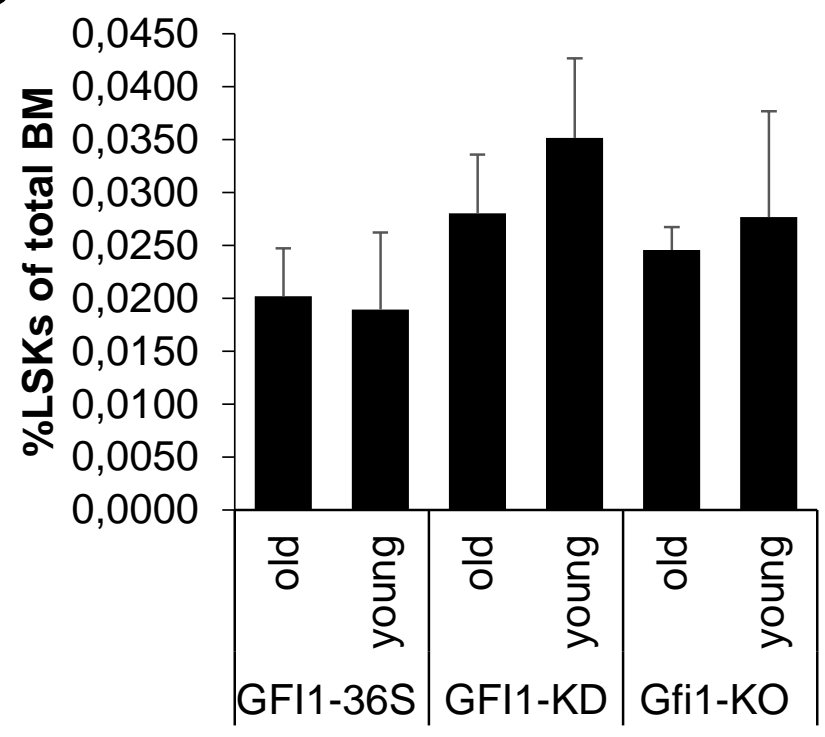


Supplementary figure 2

A Gfi1-wt + GFI1-KD

-CD45.2+ —CD45.2-

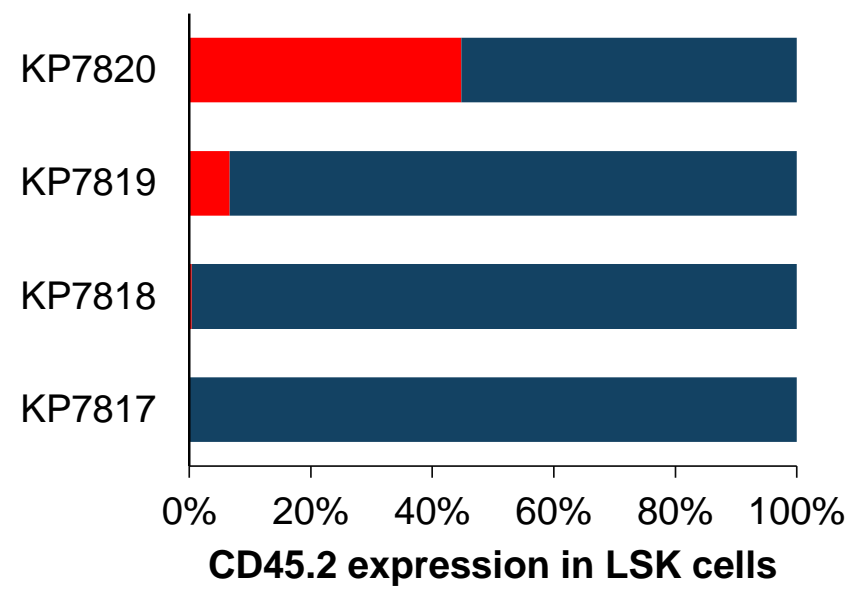

B Gfi1-wt + Gfi1-Ko

- CD45.2+ = CD45.2-

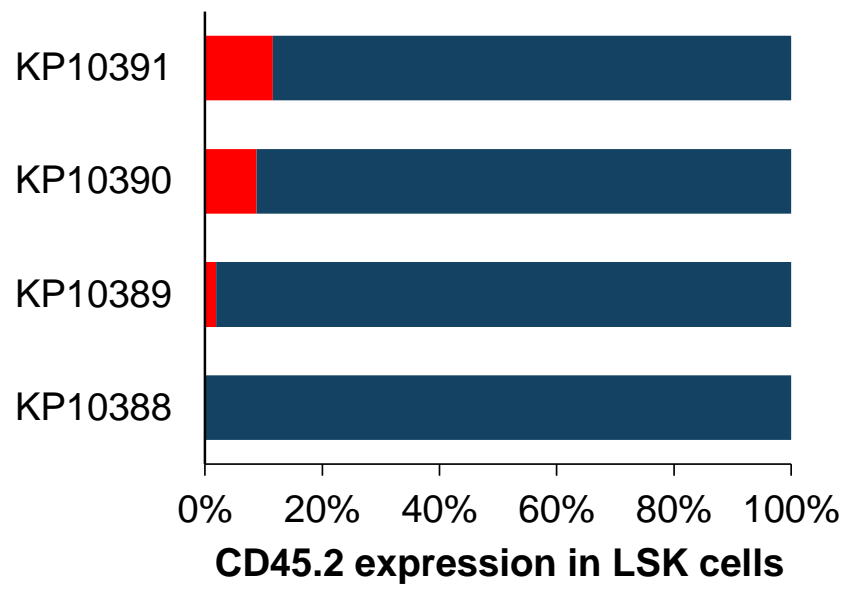

